\title{
VANISHING INSULIN REQUIREMENTS IN A PATIENT WITH TYPE 1 DIABETES
}

\author{
Ravi Bachuwar, Dinesh Nagi: Edna Coates Diabetic Centre Pinderfields General Hospitals, Mid Yorkshire NHS \\ Trust, Wakefield, UK
}

Introduction: We describe a case of a 31 year lady who had Type 1 Diabetes and started experiencing episodes of spontaneous hypoglycaemia which needed a dramatic reduction in her insulin requirements. This was due to an uncommon endocrine disorder which developed following her last pregnancy. We discuss the possible differential diagnoses of this uncommon presentation.

Clinical Presentation: This patient was diagnosed to have Type 1 diabetes and went through a successful pregnancy and delivered a healthy baby on $23 / 5 / 2015$. She presented in February 2016 with recurrent episodes of symptomatic and documented hypoglycaemia. Her insulin requirement for basal insulin fell from 36 units to 12 units / day and she had to reduce her meal related bolus insulin to 0.5 unit for every 10 gm of carbohydrates. Further questioning revealed a difficult labour, forceps delivery, intrapartum haemorrhage which needed blood transfusion. She did not breast feed and did not resume her periods post- partum.

Clinical examination showed that she was grossly hypothyroid with classic slow relaxing ankle jerks. Her blood pressure lying and standing was $123 / 88 \mathrm{mmHg}$ and $135 / 84 \mathrm{mmHg}$ and weight $81.45 \mathrm{~kg}$. She had no skin pigmentation and had no other features of Addison's disease. She denied any headaches and visual symptoms. Visual fields were full to confrontation and she had no other obvious endocrinopathies. She was commenced on Levothyroxine and Hydrocortisone treatment in the clinic pending further Endocrine assessment.

\section{Endocrine Investigations}

\begin{tabular}{|c|c|}
\hline & \\
\hline & asal Cortisol ( $\mathrm{nmol} / \mathrm{L})$ \\
\hline $\begin{array}{ll}\text { TT4 } & \text { (Normal 11-26 pmol/l) } \\
\text { =T3 } & \text { (Normal 31-6.8 } 1 \text { mol/l) }\end{array}$ & $<1.0$ \\
\hline & $\mathrm{SH} \quad$ (Normal 0.29-4.2 mU/L \\
\hline & Prolactin (Normal 0-550 miu/L) \\
\hline \multirow{4}{*}{$\begin{array}{l}\text { FSH/LH (Normal IU/L) } \\
\text { IGF-1 (Normal 14.6-39.6 nmol/l) } \\
\text { Pituitary Antibody } \\
\text { Short Svnacthen Test (SST) }\end{array}$} & $6.4 / 3.4$ \\
\hline & $<3.2 \mathrm{nmol} / \mathrm{L}$ \\
\hline & not tested \\
\hline & Short Synacthen Test (SST) \\
\hline \multirow{2}{*}{$\begin{array}{r}0 \mathrm{~min}^{\prime} \\
30 \mathrm{~min}^{\prime}\end{array}$} & 71 \\
\hline & 233 \\
\hline
\end{tabular}

Discussion: Sheehan Syndrome was originally described by Harold Sheehan in 1937, but has become a rather uncommon entity due to improvements in obstetric care both during pregnancy and labour. However, we need to be aware of this possibility in anybody in childbearing age who present with any suspicion of hypopituitarism post partum and a detailed history during labour is warranted. Other lessons to be learnt is the major drop in insulin requirement in a patient with Type 1 diabetes should a prompt look for other medical diagnosis, which includes hypopituitarism of any aetiology but also other causes especially Autoimmune hypophysitis, coeliac disease and malabsorption due to any other cause.

Our patient has responded well to treatment with Thyroxine and Hydrocortisone and is under long term follow up.

\section{MRI showing empty sella}

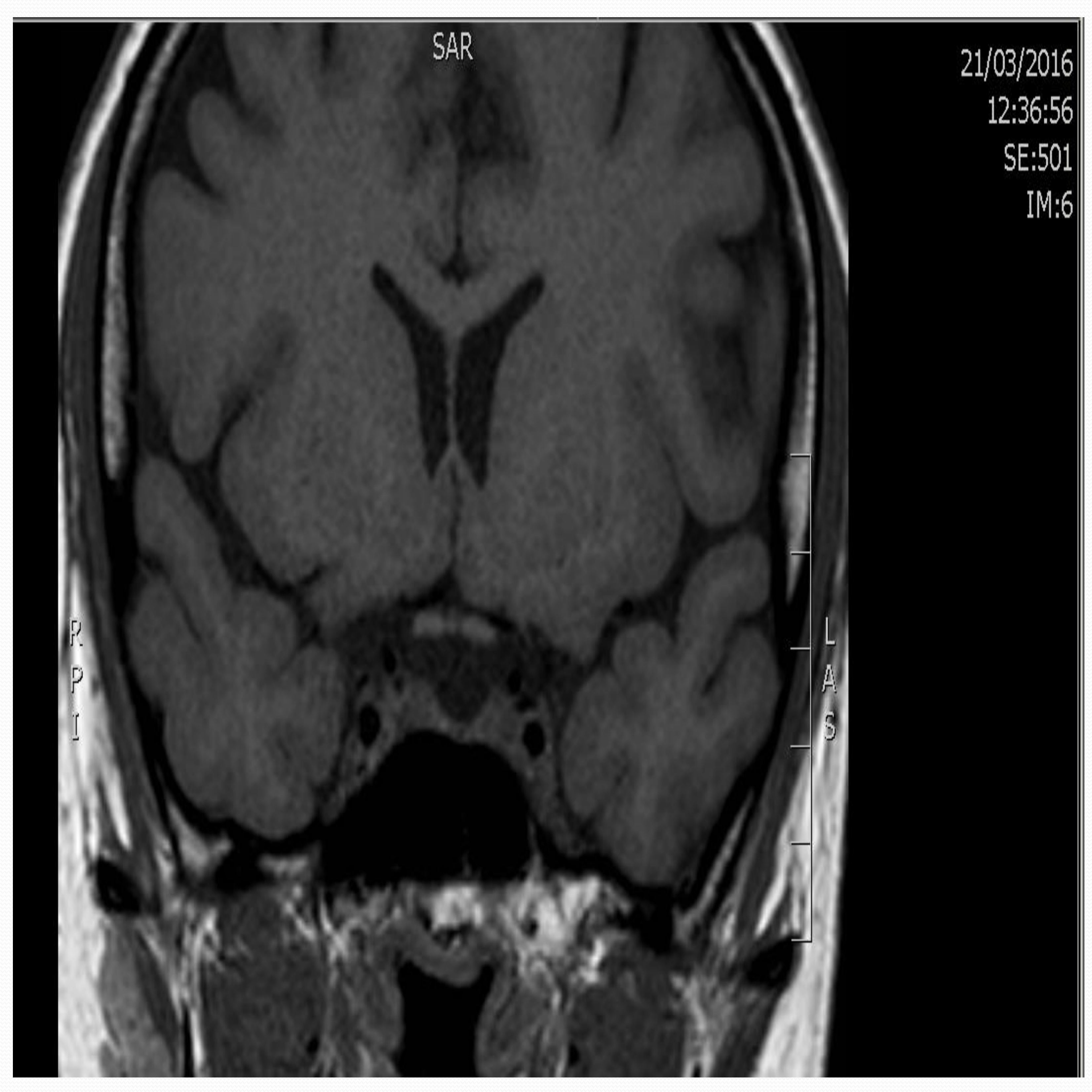

